\title{
Massa Valvar Mitral em Paciente com Suspeita de Lúpus Sistêmico: Tumor, Endocardite ou Ambos?
}

\author{
Mitral Valve Mass in a Patient Suspected of Systemic Lupus: Tumor, Endocarditis or Both?
}

\author{
Thiago Sant'Anna Coutinho, ${ }^{10}$ Bárbara Cristina Rodrigues de Almeida, ${ }^{1}$ Guilherme Dalcol Torres de Amorim, ${ }^{1}$ \\ Monica Zappa, ${ }^{1}$ Clara Weksler, ${ }^{1}$ Cristiane da Cruz Lamas ${ }^{1,2,3}{ }^{1}$ \\ Instituto Nacional de Cardiologia, ${ }^{1}$ Rio de Janeiro, RJ - Brasil \\ Instituto Nacional de Infectologia Evandro Chagas - Centro Hospitalar, ${ }^{2}$ Rio de Janeiro, RJ - Brasil \\ Universidade do Grande Rio, ${ }^{3}$ Rio de Janeiro, $R J$ - Brasil
}

\section{Resumo}

Apresentamos o relato de caso de uma paciente com mixoma valvar mitral infectado e uma revisão da literatura sobre o assunto. Uma mulher de 33 anos apresentou histórico de febre e dispneia com evolução de alguns dias. Na hospitalização, ela apresentava uma síndrome semelhante ao lúpus, com hemoculturas positivas para Haemophilus spp. O ecocardiograma revelou uma massa gigante envolvendo ambos os folhetos mitrais associada à regurgitação grave, necessitando de troca valvar mitral biológica. A microscopia revelou mixoma infectado e a paciente recebeu alta assintomática após o término da antibioticoterapia. Ela apresentou bons resultados no seguimento. Este é o sexto caso de mixoma valvar mitral infectado relatado na literatura e o terceiro caso de mixoma cardíaco infectado pelo grupo HACEK. Devido à alta incidência de eventos embólicos, a antibioticoterapia precoce aliada à pronta intervenção cirúrgica são decisivos para a redução da morbimortalidade. O tempo para o diagnóstico foi muito mais breve do que o geralmente relatado em casos de endocardite por HACEK. A troca valvar foi a intervenção mais comum e todos os pacientes em relatos de caso anteriores apresentaram bons resultados no seguimento.

\section{Introdução}

Os mixomas de válvula cardíaca são extremamente raros. ${ }^{1}$ A tríade de sintomas constitucionais, obstrutivos e embólicos torna o diagnóstico diferencial com endocardite desafiador. Excepcionalmente, os próprios mixomas podem estar infectados.

\section{Métodos}

É relatado o caso de uma paciente com mixoma valvar mitral infectado por Haemophilus spp. Uma busca nas bases

\section{Palavras-chave}

Valva Mitral/cirurgia; Valva Mitral/patologia; Diagnóstico por Imagem; Ecocardiografia; Ressonância Magnética; Mixoma Infectado; Endocardite; Lupus Eritematoso; Neoplasia Cardíaca.

Correspondência: Thiago Sant'Anna Coutinho •

Instituto Nacional de Cardiologia - Rua das Laranjeiras, 374. CEP 22240-

006, Laranjeiras, RJ - Brasil

E-mail: thiagocoutinho1989@gmail.com

Artigo recebido em 17/03/2020, revisado em 15/04/2020, aceita em 15/04/2020

DOI: https://doi.org/10.36660/abc.20200206 de dados Medline e Lilacs foi realizada desde a primeira publicação sobre o tema até 2019 para fins epidemiológicos.

\section{Resultados}

Mulher de 33 anos, previamente hígida, apresentou em dezembro de 2017 dispneia progressiva, febre alta, sudorese noturna e perda de peso. Após um mês de evolução, ela foi internada em um hospital geral em franca insuficiência respiratória e choque séptico com infiltrados alveolares difusos, icterícia, hemoptoicos e petéquias nos membros inferiores. Ela foi intubada e precisou de suporte hemodinâmico. Um leve sopro sistólico mitral foi identificado à ausculta do precórdio. Havia leucocitose acentuada com desvio à esquerda, plaquetopenia, disfunção hepática e renal associada à proteinúria subnefrótica e consumo de complemento. O resultado dos anticorpos antinucleares foi de 1/80, apesar dos níveis normais de antiDNA de fita dupla, anti-SM e anti-PR3. Após a administração de ceftriaxona, ela melhorou clinicamente. Febre amarela, dengue, Chikungunya, leptospirose, HIV e hepatites virais foram descartados. As hemoculturas foram positivas para Haemophilus spp. em todas as seis amostras coletadas. O ecocardiograma transtorácico (ETT) demonstrou uma massa ecogênica amorfa com superfície irregular e alguns elementos móveis que envolviam ambos os folhetos da válvula mitral, medindo $20 \times 17$ $\mathrm{mm}$ no folheto anterior e $19 \mathrm{~mm}$ em seu maior diâmetro nos folhetos posteriores, resultando em regurgitação grave por flail mitral e perfuração (Figura 1). A ressonância magnética mostrou pequenos abscessos esplênicos, tratados de maneira conservadora. Um aneurisma micótico não-complicado da artéria cerebral média esquerda foi tratado por embolização percutânea. Trinta dias após a hospitalização, ela foi submetida à substituição da válvula mitral com sucesso por uma prótese valvar biológica Sorin ${ }^{\circledR}$ tamanho $29 \mathrm{~mm}$ e após extensa ressecção do tumor. Foi evidenciada regurgitação aórtica moderada devido a lesão da fibrosa intervalvar mitroaórtica e retração da cúspide não coronariana, tratada de maneira conservadora. $\mathrm{O}$ exame patológico confirmou a presença de um mixoma valvar mitral infectado (Figura 1). A paciente completou 28 dias de ceftriaxona e gentamicina, recebendo alta hospitalar assintomática. No seguimento de um ano, não havia evidência de recorrência e constatava-se somente regurgitação aórtica leve. Mixomas infectados apresentam maior risco de eventos embólicos, embora as manifestações clínicas sejam indistinguíveis de tumores não infectados. ${ }^{2}$ O presente caso parece ser o sexto de mixoma valvar mitral infectado relatado na literatura, preenchendo critérios definitivos para o diagnóstico, e o terceiro causado por um 


\section{Imagem}
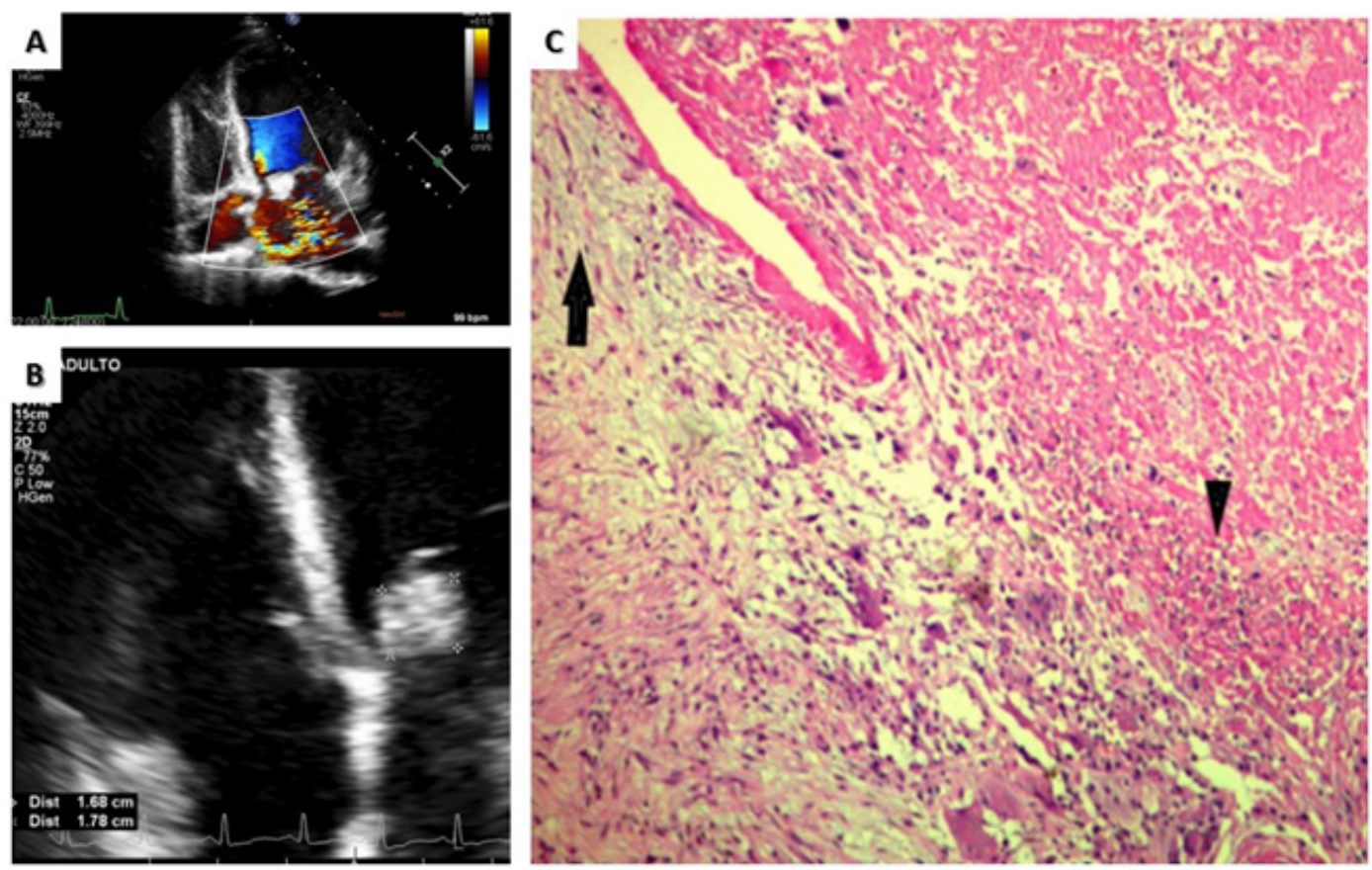

Figura 1 - A) Ecocardiografia com corte apical de quatro câmaras demonstrando insuficiência mitral grave. B) Ecocardiografia com corte apical de quatro câmaras. C) Coloração com hematoxilina-eosina 40x, o mixoma é visto na área azul formada por células estreladas em um estroma mixóide (seta), com infiltração de neutrófilos e necrose (ponta de seta).

microrganismo do grupo HACEK (Tabela 1). ${ }^{3-8}$ Dos 64 mixomas valvares mitrais publicados de 2006 a 2012, os sintomas eram cardiovasculares em $36,7 \% ; 9,5$ a $21,6 \%$ dos mixomas da válvula mitral foram submetidos à troca valvar e o tempo do diagnóstico até a cirurgia variou de algumas horas a 42 dias. ${ }^{2,9}$ A mortalidade operatória e geral foi relatada como sendo, respectivamente, 2,6 a 3\% e 5,1 a 21\%. ${ }^{2,10}$ Na presente série, a maioria dos pacientes apresentou insuficiência cardíaca sintomática, foram submetidos à troca valvar mitral e todos apresentaram bons resultados no seguimento.

\section{Conclusão}

Relatamos um inusitado caso de mixoma de válvula mitral infectado pelo grupo HACEK e complicado por êmbolos sépticos e manifestações imunomediadas. Ao revisar a literatura, descobrimos que a válvula mitral foi mais gravemente danificada pelo tumor infectado comparado aos tumores não-infectados e outros casos de endocardite por HACEK, levando a uma taxa maior de sintomas cardiovasculares e menor tempo para o diagnóstico. Apesar da cirurgia extensa com maior incidência de substituição da válvula em um ambiente de urgência, os pacientes apresentaram bons resultados no seguimento.

\section{Contribuição dos Autores}

Concepção e desenho da pesquisa: Coutinho TS, Amorim GDT, Lamas CC; Obtenção de dados: Coutinho TS, Amorim
GDT, Zappa M, Weksler C, Lamas CC; Análise e interpretação dos dados: Coutinho TS, Almeida BCR, Amorim GDT, Zappa M, Weksler C, Lamas CC; Obtenção de financiamento: Lamas CC; Redação do manuscrito: Coutinho TS, Almeida BCR, Zappa M, Lamas CC; Revisão crítica do manuscrito quanto ao conteúdo intelectual importante: Amorim GDT, Weksler C, Lamas CC.

\section{Potencial Conflito de Interesses}

Declaro não haver conflito de interesses pertinentes.

\section{Fontes de Financiamento}

O presente estudo foi financiado pela Fundação de Amparo à Pesquisa do Estado do Rio de Janeiro (grant \# E26/202.782/2015).

\section{Vinculação Acadêmica}

Não há vinculação deste estudo a programas de pós-graduação.

\section{Aprovação Ética e Consentimento Informado}

Este estudo foi aprovado pelo Comitê de Ética do Instituto Nacional de Cardiologia - INC sob o número de protocolo 3.777.454. Todos os procedimentos envolvidos nesse estudo estão de acordo com a Declaração de Helsinki de 1975, atualizada em 2013. O consentimento informado foi obtido de todos os participantes incluídos no estudo. 
Imagem

Tabela 1 - Mixomas valvares mitrais infectados descritos nas bases de dados MEDLINE e LILACS

\begin{tabular}{|c|c|c|c|c|c|c|c|c|c|}
\hline Ref. & Autor & $\begin{array}{l}\text { Anol } \\
\text { País }\end{array}$ & $\begin{array}{l}\text { Sexo/ } \\
\text { Idade } \\
\text { (anos) }\end{array}$ & $\begin{array}{c}\text { Micro- } \\
\text { organismo }\end{array}$ & Diagnóstico & Apresentação & $\begin{array}{l}\text { Localização/ } \\
\text { Cirurgia }\end{array}$ & $\begin{array}{c}\text { Complicações } \\
\text { pós-operatórias }\end{array}$ & Desfecho \\
\hline PR & Coutinho & $\begin{array}{l}2020 \\
\text { Brasil }\end{array}$ & $F / 33$ & $\begin{array}{c}\text { Haemophilus } \\
\text { spp. }\end{array}$ & $\begin{array}{c}\text { Eco } \\
\text { Critérios } \\
\text { definitivos }\end{array}$ & $\begin{array}{c}\text { Sintomas } \\
\text { constitucionais } \\
\text { Choque séptico } \\
\text { e insuficiência } \\
\text { respiratória } \\
\text { Regurgitação } \\
\text { mitral grave } \\
\text { Abscesso esplênico } \\
\text { Aneurisma micótico }\end{array}$ & $\begin{array}{c}\text { Folheto anterior } \\
\text { Tumor } 20 \times 17 \mathrm{~mm} \\
\text { Ressecção do tumor } \\
\text { e troca valvar mitral } \\
\text { biológica }\end{array}$ & $\begin{array}{l}\text { Flutter atrial } \\
\text { (imediata) } \\
\text { Regurgitação } \\
\text { aórtica } \\
\text { moderada a } \\
\text { grave } \\
\text { (tardia) }\end{array}$ & $\begin{array}{l}\text { Sobreviveu } \\
\text { NYHAI } \\
\text { Sem } \\
\text { recorrência } \\
\text { Seguimento } \\
\text { de } 1 \text { ano }\end{array}$ \\
\hline (8) & Ghazi & $\begin{array}{l}1988 \\
\text { Reino } \\
\text { Unido }\end{array}$ & $\mathrm{F} / 17$ & $\begin{array}{l}\text { Haemophilus } \\
\text { parainfluenzae }\end{array}$ & $\begin{array}{c}\text { Eco } \\
\text { Critérios } \\
\text { definitivos }\end{array}$ & $\begin{array}{c}\text { Sintomas } \\
\text { constitucionais } \\
\text { Vômito, diarreia } \\
\text { e dor abdominal } \\
\text { Sepse } \\
\text { Insuficiência mitral }\end{array}$ & $\begin{array}{l}\text { Folheto posterior } \\
\text { Tumor de 10mm } \\
\text { Ressecção do tumor } \\
\text { e anuloplastia }\end{array}$ & $\begin{array}{l}\text { Sem } \\
\text { intercorrências }\end{array}$ & $\begin{array}{l}\text { Sobreviveu } \\
\text { NYHAI } \\
\text { Sem } \\
\text { recorrência } \\
\text { Seguimento } \\
\text { de } 9 \text { meses }\end{array}$ \\
\hline (7) & Mrozinski & $\begin{array}{c}1997 \\
\text { Polônia }\end{array}$ & $\mathrm{F} / 4$ & $\begin{array}{c}\text { Staphylococcus } \\
\text { aureus }\end{array}$ & $\begin{array}{c}\text { Eco } \\
\text { Critérios } \\
\text { definitivos }\end{array}$ & $\begin{array}{c}\text { Sintomas } \\
\text { constitucionais } \\
\text { Insuficiência } \\
\text { cardíaca aguda } \\
\text { Regurgitação mitral } \\
\text { grave }\end{array}$ & $\begin{array}{l}\text { Ambos os folhetos } \\
\text { Tumor } 30 \mathrm{~mm} \\
\text { Ressecção do } \\
\text { tumor e substituição } \\
\text { por válvula mitral } \\
\text { mecânica }\end{array}$ & $\begin{array}{c}\text { Sem } \\
\text { intercorrências }\end{array}$ & $\begin{array}{l}\text { Sobreviveu } \\
\text { NYHAI } \\
\text { Sem } \\
\text { recorrência } \\
\text { Tempo de } \\
\text { seguimento } \\
\text { não está } \\
\text { claro }\end{array}$ \\
\hline (6) & Toda & $\begin{array}{l}1999 \\
\text { Japão }\end{array}$ & $\mathrm{M} / 20$ & $\begin{array}{l}\text { Hemoculturas } \\
\text { negativas } \\
\text { Bactérias } \\
\text { observadas na } \\
\text { patologia }\end{array}$ & $\begin{array}{c}\text { Eco } \\
\text { Critérios } \\
\text { definitivos }\end{array}$ & $\begin{array}{c}\text { Sintomas } \\
\text { constitucionais } \\
\text { Síncope } \\
\text { Oclusão arterial aguda } \\
\text { de membro } \\
\text { Regurgitação mitral } \\
\text { moderada }\end{array}$ & $\begin{array}{l}\text { Folheto posterior } \\
\text { Tumor } 20 \mathrm{~mm} \\
\text { Ressecção de tumor } \\
\text { e cordas, troca valvar } \\
\text { mitral mecânica }\end{array}$ & $\begin{array}{c}\text { Sem } \\
\text { intercorrências }\end{array}$ & $\begin{array}{l}\text { Sobreviveu } \\
\text { NYHAI } \\
\text { Sem } \\
\text { recorrência } \\
\text { Seguimento } \\
\text { de } 2 \text { anos }\end{array}$ \\
\hline (5) & Liu & $\begin{array}{l}2005 \\
\text { China }\end{array}$ & $F / 12$ & $\begin{array}{l}\text { Neisseria } \\
\text { lactamica }\end{array}$ & $\begin{array}{c}\text { Eco } \\
\text { Critérios } \\
\text { definitivos }\end{array}$ & $\begin{array}{l}\text { Sintomas } \\
\text { constitucionais } \\
\text { Insuficiência } \\
\text { cardíaca aguda } \\
\text { Regurgitação } \\
\text { mitral grave }\end{array}$ & $\begin{array}{l}\text { Folheto anterior } \\
\text { Tumor de } 35 \times 25 \mathrm{~mm} \\
\text { Ressecção do tumor } \\
\text { e músculo papilar, } \\
\text { troca valvar mitral } \\
\text { mecânica e cirurgia } \\
\text { de revascularização } \\
\text { do miocárdio }\end{array}$ & $\begin{array}{c}\text { Sem } \\
\text { intercorrências }\end{array}$ & $\begin{array}{l}\text { Sobreviveu } \\
\text { Sem dados } \\
\text { sobre a } \\
\text { classe } \\
\text { funcional } \\
\text { Sem } \\
\text { recorrência } \\
\text { Seguimento } \\
\text { de } 6 \text { anos }\end{array}$ \\
\hline (4) & Guler & $\begin{array}{c}2007 \\
\text { Turquia }\end{array}$ & $\mathrm{F} / 12$ & $\begin{array}{c}\text { Staphylococcus } \\
\text { aureus }\end{array}$ & $\begin{array}{c}\text { Eco } \\
\text { Critérios } \\
\text { definitivos }\end{array}$ & $\begin{array}{c}\text { Início agudo de febre, } \\
\text { suor e fadiga } \\
\text { Sepse } \\
\text { Regurgitação } \\
\text { mitral leve }\end{array}$ & $\begin{array}{l}\text { Folheto anterior } \\
\text { Tumor } 29 \times 18 \mathrm{~mm} \\
\text { Ressecção de tumor }\end{array}$ & $\begin{array}{c}\text { Rotura de } \\
\text { aneurisma } \\
\text { sacular de aorta } \\
\text { abdominal e } \\
\text { infarto renal } \\
\text { bilateral (tardio) }\end{array}$ & $\begin{array}{l}\text { Sobreviveu } \\
\text { Sem dados } \\
\text { sobre a } \\
\text { classe } \\
\text { funcional } \\
\text { Sem } \\
\text { recorrência } \\
\text { Seguimento } \\
\text { de } 6 \text { meses }\end{array}$ \\
\hline
\end{tabular}

PR: presente relato; F: feminino; M: masculino; Eco: ecocardiograma; NYHA: classe funcional segundo a New York Heart Association 


\section{Referências}

1. Yuan SM. Mitral valve myxoma: clinical features, current diagnostic approaches, and surgical management. Cardiol J. 2012;19(1):105-9.

2. Yuan S-M. Infected cardiac myxoma: an updated review. Braz J Cardiovasc Surg. 2015;30(5):571-8.

3. Bernstein JM, Leasure W, Buel A. Getting to the heart of the matter. Skinmed. 2007;6(6):290-2.

4. Guler N, Ozkara C, Kaya Y, Saglam E. Ruptured abdominal aortic aneurysm after resection of an infected cardiac myxoma. Texas Heart Inst J. 2007;34(2):233.

5. Liu YL, Liu XH, Cai KH. Cardiac valve myxomas with infected endocarditis in the youngerly. Zhonghua bing li xue za zhi= Chin J Pathol. 2005;34(10):695-6.
6. Toda R, Moriyama Y, Shiota K, Toyohira H, Taira A. Myxoma of mitral valve associated with infective endocarditis. Japan J Thorac Cardiovasc Surg. 1999;47(6):285-7.

7. Mroziski B, Siwiska A, Górzna H, Szelagowicz B, Maciejewski J. Case of the month: a 4-year-old girl with cardiac insufficiency and intermittent fever. Eur J Pediatr. 1997;156(2):153-4.

8. Ghazi F, Sterba R, Moodie DS, Gill CC, Ratliff NB. Myxoma of the mitral valve associated with Hemophilus parainfluenza bacteremia. Clev Clin J Med. 1988;55(5):470-2.

9. Kretz J-G, Valentin P, Petit H, Popescu S, Edah-Tally S, Massard G. Clinical presentation and treatment options for mitral valve myxoma. Ann Thorac Surg. 1997;64(3):872-7.

10. Revankar SG, Clark RA. Infected cardiac myxoma. Case report and literature review. Medicine (Baltimore). 1998;77(5):337-44. 